\title{
Maximax rearrangement optimization related to a homogeneous Dirichlet problem
}

Received: 26 June 2012 / Accepted: 1 July 2013 / Published online: 19 July 2013

(C) The Author(s) 2013. This article is published with open access at Springerlink.com

\begin{abstract}
In this paper we investigate a maximax optimization problem related to a homogeneous Dirichlet problem in two classes of rearrangements. We prove existence and representation of the maximizers.
\end{abstract}

Mathematics Subject Classification 35J20 • 49J20

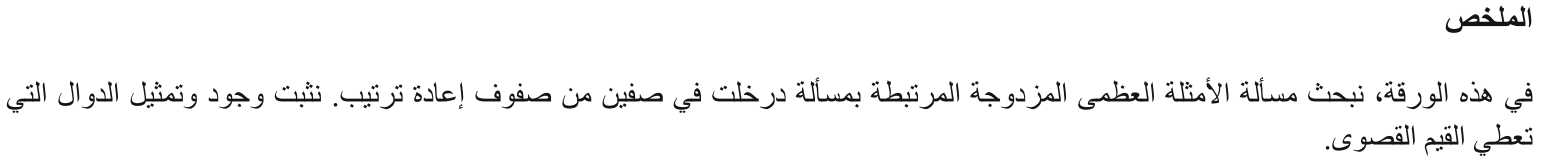

\section{Introduction}

In this paper we investigate an optimization problem of maximax type related to the following boundary value problem

$$
-\Delta u+h(x) u=f(x) \text { in } \Omega \text {, and } u=0 \text { on } \partial \Omega .
$$

Here, $\Omega$ is a smooth bounded domain in $\mathbb{R}^{N}, h$ a non-negative function in $L^{\infty}(\Omega)$, and $f$ a function in $L^{2}(\Omega)$. It is well-known that (1) has a unique weak solution in $H_{0}^{1}(\Omega)$; see [4]. We denote the solution by $u_{f h}$ to emphasize on its dependence on $f$ and $h$. The problem (1) has been widely used to model many physical phenomena, but the one we briefly explain here is related to the bending of an elastic membrane. Suppose $\Omega$ is a planar region occupied by an elastic membrane fixed around the boundary. The membrane is assumed to be non-isotropic; that is, it is made of several materials with different densities, and hence the presence of the function $h$ in (1) is justified. Further, we assume the membrane is subject to a vertical force such as a load distribution, and this justifies the use of a function like $f$ on the right hand side of the differential equation in (1). Finally, the solution $u_{f h}$ denotes the displacement (bending) of the membrane at various points of $\Omega$.

We now proceed to introduce the optimization problem that we are interested in. Note that the setting is going to be done in any dimension in contrast to the above physical description of (1) in dimension two. We first define a quantity as follows

$$
J(f, h)=\int_{\Omega} f u_{f h} \mathrm{~d} x .
$$

M. Zivari-Rezapour ( $\varangle)$

Department of Mathematics, Faculty of Mathematical Sciences and Computer, Shahid Chamran University,

Golestan Blvd., Ahvaz, Iran

E-mail: mzivari@scu.ac.ir 
Recalling the variational formulation of $u_{f h}$, see the next section, confirms the functional $J: L^{2}(\Omega) \times$ $L^{\infty}(\Omega) \rightarrow \mathbb{R}$ is well defined. Our interest is in the following maximax optimization problem

$$
\max _{h \in \mathcal{R}\left[h_{0}\right]} \max _{f \in \mathcal{R}\left[f_{0}\right]} J(f, h),
$$

where $h_{0}$ and $f_{0}$ satisfy the conditions mentioned above pertaining to the two functions $h$ and $f$ in (1). Moreover, $\mathcal{R}\left[h_{0}\right]$ and $\mathcal{R}\left[f_{0}\right]$ indicate two rearrangement classes generated by $h_{0}$ and $f_{0}$, respectively; see the next section for precise definition. The main result of this paper is Theorem 4.2, where we prove (2) is solvable, contingent to $f_{0}$ and $h_{0}$ satisfying a technical condition. By solvability of (2), we mean existence of a pair $(\hat{f}, \hat{h}) \in \mathcal{R}\left[f_{0}\right] \times \mathcal{R}\left[h_{0}\right]$ such that

$$
J(\hat{f}, \hat{h})=\max _{h \in \mathcal{R}\left[h_{0}\right]} \max _{f \in \mathcal{R}\left[f_{0}\right]} J(f, h) .
$$

Optimization problems where the admissible set is a rearrangement class or a subset of that have become quite popular in recent years; see for example [5-13] and references therein. However, the one which is discussed here is unique in the sense that the admissible set is a cross product of two rearrangement classes. This problem is physically interesting as well. Let us go back to the membrane problem described above. In that setting, the optimization problem (2) is asking for the best design of the membrane (the function $\hat{h}$ ), and the optimal load distribution (the function $\hat{f}$ ) which maximizes the quantity $J$.

\section{Preliminaries}

In this section we collect some well-known results.

Let $\Omega$ be a smooth bounded domain in $\mathbb{R}^{N}$. For any $E \subset \Omega$ we denote the $N$-dimensional Lebesgue measure of $E$, by $\mathcal{L}_{N}(E)$. Let $f, g: \Omega \rightarrow \mathbb{R}$ be two measurable functions. We say $f$ and $g$ are rearrangement of each other provided

$$
\mathcal{L}_{N}(\{x \in \Omega: f(x) \geq c\})=\mathcal{L}_{N}(\{x \in \Omega: g(x) \geq c\}),
$$

for all $c \in \mathbb{R}$. It is well known that if $f \in L^{p}(\Omega), 1 \leq p \leq \infty$, and $g$ be a rearrangement of $f$, then $g \in L^{p}(\Omega)$ and in fact $\|f\|_{p}=\|g\|_{p}$, where $\|\cdot\|_{p}$ denotes the standard norm of $L^{p}(\Omega)$. The rearrangement class by $f$ is denoted $\mathcal{R}[f]$, which comprises all functions that are rearrangements of $f$. The readers can study [2,3] for more results about rearrangements of functions.

We say $u \in H_{0}^{1}(\Omega)$ is the weak solution of (1) whenever

$$
\int_{\Omega} \nabla u \cdot \nabla v \mathrm{~d} x+\int_{\Omega} h u v \mathrm{~d} x=\int_{\Omega} f v \mathrm{~d} x, \quad \text { for all } v \in H_{0}^{1}(\Omega) .
$$

It is well known that $u$, the unique weak solution of (1), satisfies the following variational problem

$$
\begin{aligned}
\int_{\Omega} f u \mathrm{~d} x & =\int_{\Omega}|\nabla u|^{2} \mathrm{~d} x+\int_{\Omega} h u^{2} \mathrm{~d} x \\
& =\max _{w \in H_{0}^{1}(\Omega)}\left\{2 \int_{\Omega} f w \mathrm{~d} x-\int_{\Omega} h w^{2} \mathrm{~d} x-\int_{\Omega}|\nabla w|^{2} \mathrm{~d} x\right\} .
\end{aligned}
$$

We now collect some useful lemmas to be applied later.

Lemma 2.1 ([3]) Let $1 \leq p \leq \infty$ and $q$ be the conjugate exponent of $p$. Let $f_{0} \in L^{p}(\Omega)$. Then,

(i) $\overline{\mathcal{R}}\left[f_{0}\right]$, the weak closure of $\mathcal{R}\left[f_{0}\right]$ in $L^{p}(\Omega)$, is compact with respect to $L^{q}$-topology on $L^{p}(\Omega)$.

(ii) $\overline{\mathcal{R}}\left[f_{0}\right]$ is convex.

Remark 2.2 $L^{q}$-topology on $L^{p}(\Omega)$ is the weak topology for $1 \leq p<\infty$ and is the weak* topology for $p=\infty$. 
Lemma 2.3 ([3]) Let $f_{0}: \Omega \rightarrow \mathbb{R}$ and $g: \Omega \rightarrow \mathbb{R}$ be two measurable functions. If every level set of $g$ has measure zero, then there exists an increasing function $\varphi$ such that $\varphi \circ g \in \mathcal{R}\left[f_{0}\right]$. Furthermore, there exists a decreasing function $\psi$ such that $\psi \circ g \in \mathcal{R}\left[f_{0}\right]$.

Lemma 2.4 ([3]) Let $1 \leq p \leq \infty$ and $q$ be the conjugate exponent of $p$. Let $f_{0} \in L^{p}(\Omega)$ and $g \in L^{q}(\Omega)$. Thus

(i) If there is an increasing function $\varphi$ such that $\varphi \circ g \in \mathcal{R}\left[f_{0}\right]$ then

$$
\int_{\Omega} f g \mathrm{~d} x \leq \int_{\Omega}(\varphi \circ g) g \mathrm{~d} x, \text { for all } f \in \mathcal{R}\left[f_{0}\right] .
$$

(ii) If there is a decreasing function $\psi$ such that $\psi \circ g \in \mathcal{R}\left[f_{0}\right]$ then

$$
\int_{\Omega} f g \mathrm{~d} x \geq \int_{\Omega}(\psi \circ g) g \mathrm{~d} x, \text { for all } f \in \mathcal{R}\left[f_{0}\right] .
$$

Lemma 2.5 ([2]) Let $1 \leq p \leq \infty$ and $q$ be the conjugate exponent of $p$. Let $f_{0} \in L^{p}(\Omega)$ and $\Psi: L^{p}(\Omega) \rightarrow \mathbb{R}$ be convex.

(i) Suppose that $\Psi$ is sequentially continuous in the $L^{q}$-topology on $L^{p}(\Omega)$. Then $\Psi$ attains a maximum value relative to $\mathcal{R}\left[f_{0}\right]$.

(ii) Suppose that $\Psi$ is strictly convex, that $f^{*}$ is a maximizer for $\Psi$ relative to $\mathcal{R}\left[f_{0}\right]$ and that $g$ is a member of sub-gradient of $\Psi$ at $f^{*}$. Then $f^{*}=\varphi \circ g$ almost everywhere in $\Omega$ for some increasing function $\varphi$.

\section{The inverse of $-\Delta+h I$}

Let $h$ be a non-negative function in $L^{\infty}(\Omega)$ and $I$ stand for identity operator. Let $K_{h}: L^{2}(\Omega) \rightarrow H_{0}^{1}(\Omega)$ denote the inverse of $-\Delta+h I$ with Dirichlet homogeneous boundary conditions on $\partial \Omega$. Let $f \in L^{2}(\Omega)$, thus $K_{h}(f)$ is the unique weak solution of problem (1) corresponding to $f$ and $h$. In the following, we prove some properties of $K_{h}$.

(P1) $K_{h}$ is a bounded linear operator.

Proof From (4) and Hölder's inequality we infer that

$$
\int_{\Omega} \mid \nabla\left(\left.K_{h}(f)\right|^{2} \mathrm{~d} x \leq \int_{\Omega} f K_{h}(f) \mathrm{d} x \leq\|f\|_{2}\left\|K_{h}(f)\right\|_{2} .\right.
$$

By continuous imbedding of $H_{0}^{1}(\Omega)$ into $L^{2}(\Omega)$, see [1], there exists a positive constant $C$ such that

$$
\left\|K_{h}(f)\right\| \leq C\|f\|_{2} \text {. }
$$

Here, $\|\cdot\|=\left(\int_{\Omega}|\nabla(.)|^{2} \mathrm{~d} x\right)^{1 / 2}$ is equivalent norm in $H_{0}^{1}(\Omega)$. Therefore, $K_{h}$ is a bounded (continuous) linear operator.

Remark 3.1 By compact imbedding of $H_{0}^{1}(\Omega)$ into $L^{2}(\Omega)$, see [1], we infer that $K_{h}$ is a compact operator.

(P2) $K_{h}$ is symmetric; that is,

$$
\int_{\Omega} f K_{h}(g) \mathrm{d} x=\int_{\Omega} g K_{h}(f) \mathrm{d} x \text { for all } f, g \in L^{2}(\Omega) .
$$

Proof It is clear from (3).

(P3) $K_{h}$ is positive; that is, $\int_{\Omega} f K_{h}(f) \mathrm{d} x \geq 0$ for all $f \in L^{2}(\Omega)$.

Proof It is clear from (4). 


\section{Maximax optimization}

We begin with the following theorem.

Theorem 4.1 Let $h$ be a non-negative function in $L^{\infty}(\Omega)$ and $f_{0} \in L^{2}(\Omega)$. Then the maximization problem

$$
\max _{f \in \mathcal{R}\left[f_{0}\right]} J(f, h),
$$

is solvable; that is, there exists $f_{h} \in \mathcal{R}\left[f_{0}\right]$ such that

$$
J\left(f_{h}, h\right)=\max _{f \in \mathcal{R}\left[f_{0}\right]} \int_{\Omega} f K_{h}(f) \mathrm{d} x .
$$

Moreover, there exists an increasing function $\varphi$ such that $f_{h}=\varphi \circ K_{h}\left(f_{h}\right)$ almost everywhere in $\Omega$.

Proof First, we show that the functional

$$
J(f, h)=\int_{\Omega} f K_{h}(f) \mathrm{d} x
$$

is Gâteaux differentiable with respect to $f \in L^{2}(\Omega)$. Let $f, g \in L^{2}(\Omega)$. Since $K_{h}$ is symmetric, Property (P2) of $K_{h}$, we infer that

$$
\left\langle D_{1} J(f, h), g\right\rangle:=\lim _{t \rightarrow 0} \frac{J(f+t g, h)-J(f, h)}{t}=2 \int_{\Omega} g K_{h}(f) \mathrm{d} x .
$$

$J(., h)$ is weakly sequentially continuous, because $K_{h}$ is a linear compact operator; see Remark 3.1. Now, we show that $J(., h)$ is strictly convex. For any $h \in L^{\infty}(\Omega), f \in L^{2}(\Omega)$ and $w \in H_{0}^{1}(\Omega)$ define

$$
\Lambda(w, f, h):=2 \int_{\Omega} f w \mathrm{~d} x-\int_{\Omega} h w^{2} \mathrm{~d} x-\int_{\Omega}|\nabla w|^{2} \mathrm{~d} x .
$$

So, from (4) we deduce

$$
J(f, h)=\sup _{w \in H_{0}^{1}(\Omega)} \Lambda(w, f, h) .
$$

Let $t \in[0,1], h \in L^{\infty}(\Omega)$ and $f, g \in L^{2}(\Omega)$, we have

$$
\Lambda(w, t f+(1-t) g, h)=t \Lambda(w, f, h)+(1-t) \Lambda(w, g, h)
$$

thus

$$
J(t f+(1-t) g, h) \leq t J(f, h)+(1-t) J(g, h) .
$$

Hence, $J(., h)$ is convex. Suppose for some $t \in(0,1)$, we have

$$
J(t f+(1-t) g, h)=t J(f, h)+(1-t) J(g, h) .
$$

Thus,

$$
\Lambda\left(K_{h}(t f+(1-t) g), t f+(1-t) g, h\right)=t \Lambda\left(K_{h}(f), f, h\right)+(1-t) \Lambda\left(K_{h}(g), g, h\right)
$$

so

$$
\begin{aligned}
& t \Lambda\left(K_{h}(t f+(1-t) g), f, h\right)+(1-t) \Lambda\left(K_{h}(t f+(1-t) g), g, h\right) \\
& \quad=t \Lambda\left(K_{h}(f), f, h\right)+(1-t) \Lambda\left(K_{h}(g), g, h\right) .
\end{aligned}
$$

Therefore, $K_{h}(t f+(1-t) g)=K_{h}(f)=K_{h}(g)$ almost everywhere in $\Omega$. Since $K_{h}$ is linear and invertible, we deduce $f=g$ almost everywhere in $\Omega$. Therefore $J(., h)$ is strictly convex. Now by applying Lemma 2.5 we infer that there exists $f_{h} \in \mathcal{R}\left[f_{0}\right]$ such that

$$
J\left(f_{h}, h\right)=\max _{f \in \mathcal{R}\left[f_{0}\right]} J(f, h)=\int_{\Omega} f_{h} K_{h}\left(f_{h}\right) \mathrm{d} x,
$$

and $f_{h}=\varphi \circ K_{h}\left(f_{h}\right)$ almost everywhere in $\Omega$, for some increasing function $\varphi$. 
Now, we are ready to prove the following theorem.

Theorem 4.2 Let $h_{0}$ be a non-negative function in $L^{\infty}(\Omega)$ and $f_{0} \in L^{2}(\Omega)$ such that $\mathcal{L}_{N}\left(\left\{x \in \Omega:\left|f_{0}(x)\right|=\right.\right.$ $\left.\left.c h_{0}(x)\right\}\right)=0$ for all $c \geq 0$. Then the following maximax optimization problem is solvable

$$
\max _{h \in \mathcal{R}\left[h_{0}\right]} \max _{f \in \mathcal{R}\left[f_{0}\right]} J(f, h) .
$$

Proof Theorem 4.1 implies that we must prove the following optimization problem is solvable

$$
\gamma=\max _{h \in \mathcal{R}\left[h_{0}\right]} L(h)
$$

where $L(h):=J\left(f_{h}, h\right)$. From Hölder's inequality, the continuous embedding of $H_{0}^{1}(\Omega)$ into $L^{2}(\Omega)$ and inequality (5), for all $h \in \mathcal{R}\left[h_{0}\right]$ we have

$$
\begin{aligned}
|L(h)|=\left|\int_{\Omega} f_{h} K_{h}\left(f_{h}\right) \mathrm{d} x\right| & \leq\left\|f_{h}\right\|_{2}\left\|K_{h}\left(f_{h}\right)\right\|_{2} \\
& \leq C\left\|f_{h}\right\|_{2}\left\|K_{h}\left(f_{h}\right)\right\| \\
& \leq C\left\|f_{h}\right\|_{2}^{2}=C\left\|f_{0}\right\|_{2}^{2},
\end{aligned}
$$

where $C$ denotes a positive constant that can change from line to line. Thus, $L$ is bounded on $\mathcal{R}\left[h_{0}\right]$. Assume $\left\{h_{i}\right\}$ to be a maximizing sequence for the problem (7). For simplicity we set $f_{i}:=f_{h_{i}}$ and $K_{i}:=K_{h_{i}}$. From (5), we infer that $\left\{K_{i}\left(f_{i}\right)\right\}$ is a bounded sequence in $H_{0}^{1}(\Omega)$, thus there exists a subsequence, still denoted $\left\{K_{i}\left(f_{i}\right)\right\}$, that converges weakly to $v \in H_{0}^{1}(\Omega)$. The compact imbedding of $H_{0}^{1}(\Omega)$ into $L^{2}(\Omega)$ implies that $\left\{K_{i}\left(f_{i}\right)\right\}$ converges strongly to $v$ in $L^{2}(\Omega)$. Since $\left\{h_{i}\right\}$ and $\left\{f_{i}\right\}$ are bounded sequences in $\mathcal{R}\left[h_{0}\right]$ and $\mathcal{R}\left[f_{0}\right]$, respectively, by applying Lemma 2.1 , there exist subsequences of those, still denoted $\left\{h_{i}\right\}$ and $\left\{f_{i}\right\}$, and $\eta \in \overline{\mathcal{R}}\left[h_{0}\right]$ and $\xi \in \overline{\mathcal{R}}\left[f_{0}\right]$ such that $\left\{h_{i}\right\}$ converges weakly* to $\eta$ in $L^{\infty}(\Omega)$ and $\left\{f_{i}\right\}$ converges weakly to $\xi$ in $L^{2}(\Omega)$. Now by the weak lower semicontinuity of Dirichlet integral, we drive

$$
\begin{aligned}
\gamma & =\lim _{i \rightarrow \infty} L\left(h_{i}\right) \\
& =\lim _{i \rightarrow \infty}\left\{2 \int_{\Omega} f_{i} K_{i}\left(f_{i}\right) \mathrm{d} x-\int_{\Omega} h_{i} K_{i}^{2}\left(f_{i}\right) \mathrm{d} x-\int_{\Omega}\left|\nabla K_{i}\left(f_{i}\right)\right|^{2} \mathrm{~d} x\right\} \\
& \leq 2 \int_{\Omega} \xi v \mathrm{~d} x-\int_{\Omega} \eta v^{2} \mathrm{~d} x-\int_{\Omega}|\nabla v|^{2} \mathrm{~d} x .
\end{aligned}
$$

From (3), we have

$$
\int_{\Omega} \nabla K_{i}\left(f_{i}\right) \cdot \nabla w \mathrm{~d} x+\int_{\Omega} h_{i} K_{i}\left(f_{i}\right) w \mathrm{~d} x=\int_{\Omega} f_{i} w \mathrm{~d} x, \quad \text { for all } w \in H_{0}^{1}(\Omega) .
$$

Thus, when $i \rightarrow \infty$, we deduce that

$$
\int_{\Omega} \nabla v \cdot \nabla w \mathrm{~d} x+\int_{\Omega} \eta v w \mathrm{~d} x=\int_{\Omega} \xi w \mathrm{~d} x, \quad \text { for all } w \in H_{0}^{1}(\Omega) .
$$

Therefore $v$ is the weak solution of the following problem

$$
-\Delta v+\eta(x) v=\xi(x) \text { in } \Omega, \text { and } v=0 \text { on } \partial \Omega .
$$

Since $\mathcal{L}_{N}\left(\left\{x \in \Omega:\left|f_{0}(x)\right|=c h_{0}(x)\right\}\right)=0$ for all $c \geq 0$, from (9) we infer that every level set of $v$ has measure zero. So, Lemma 2.3 and Lemma 2.4 imply that there exist $\hat{f} \in \mathcal{R}\left[f_{0}\right]$ and $\hat{h} \in \mathcal{R}\left[h_{0}\right]$ such that

$$
\int_{\Omega} \xi v \mathrm{~d} x \leq \int_{\Omega} \hat{f} v \mathrm{~d} x \text { and } \int_{\Omega} \eta v^{2} \mathrm{~d} x \geq \int_{\Omega} \hat{h} v^{2} \mathrm{~d} x .
$$


Now from (8) and (10), we infer that

$$
\begin{aligned}
\gamma & \leq 2 \int_{\Omega} \hat{f} v \mathrm{~d} x-\int_{\Omega} \hat{h} v^{2} \mathrm{~d} x-\int_{\Omega}|\nabla v|^{2} \mathrm{~d} x \\
& \leq J(\hat{f}, \hat{h}) \leq J\left(f_{\hat{h}}, \hat{h}\right)=L(\hat{h}) \leq \gamma .
\end{aligned}
$$

Therefore $\gamma=L(\hat{h})=J\left(f_{\hat{h}}, \hat{h}\right)$ and this completes the proof of the theorem.

Remark 4.3 Assume that

$$
J\left(f_{\hat{h}}, \hat{h}\right)=\max _{h \in \mathcal{R}\left[h_{0}\right]} \max _{f \in \mathcal{R}\left[f_{0}\right]} J(f, h) .
$$

We set $\hat{u}=K_{\hat{h}}\left(f_{\hat{h}}\right)$. For any $h \in \mathcal{R}\left[h_{0}\right]$ we have

$$
\begin{aligned}
J\left(f_{\hat{h}}, \hat{h}\right) & =2 \int_{\Omega} f_{\hat{h}} \hat{u} \mathrm{~d} x-\int_{\Omega} \hat{h}(\hat{u})^{2} \mathrm{~d} x-\int_{\Omega}|\nabla \hat{u}|^{2} \mathrm{~d} x \\
& \geq J\left(f_{\hat{h}}, h\right) \geq 2 \int_{\Omega} f_{\hat{h}} \hat{u} \mathrm{~d} x-\int_{\Omega} h(\hat{u})^{2} \mathrm{~d} x-\int_{\Omega}|\nabla \hat{u}|^{2} \mathrm{~d} x .
\end{aligned}
$$

Thus

$$
\int_{\Omega} \hat{h}(\hat{u})^{2} \mathrm{~d} x \leq \int_{\Omega} h(\hat{u})^{2} \mathrm{~d} x \text { for all } h \in \mathcal{R}\left[h_{0}\right] .
$$

Now, by applying (11) and the similar methods that we used in Lemma 4.2 and Theorem 4.3 of [11], we infer that there exists a decreasing function $\psi$, such that $\hat{h}=\psi \circ \hat{u}$ almost everywhere in $\Omega$. Therefore, from this fact and Theorem 4.1, we deduce that

$$
J\left(\varphi\left(K_{\hat{h}}\left(f_{\hat{h}}\right)\right), \psi\left(K_{\hat{h}}\left(f_{\hat{h}}\right)\right)\right)=\max _{h \in \mathcal{R}\left[h_{0}\right]} \max _{f \in \mathcal{R}\left[f_{0}\right]} \int_{\Omega} f K_{h}(f) \mathrm{d} x .
$$

Acknowledgments I wish to thank the referees for their helpful comments. Also, I would like to express my sincere thanks to Prof. Behrouz Emamizadeh for his valuable comments on the Introduction section.

Open Access This article is distributed under the terms of the Creative Commons Attribution License which permits any use, distribution, and reproduction in any medium, provided the original author(s) and the source are credited.

\section{References}

1. Adams, R.: Sobolev Spaces. Academic Press, New York (1975)

2. Burton, G.R.: Rearrangements of functions, maximization of convex functionals, and vortex rings. Math. Ann. 276, 225-253 (1987)

3. Burton, G.R.: Variational problems on classes of rearrangements and multiple configurations for steady vortices. Ann. Inst. Henri Poincare 6, 295-319 (1989)

4. Badiale, M.; Serra, E.: Semilinear elliptic equations for beginners, existence results via the variational approach, Universitext. Springer, London (2011), ISBN: 978-0-85729-226-1

5. Cuccu, F.; Emamizadeh, B.; Porru, G.: Optimization of the first eigenvalue in problems involving the $p$-Laplacian. Proc. Am. Math. Soc. 137(5), 1677-1687 (2009)

6. Cuccu, F.; Emamizadeh, B.; Porru, G.: Optimization problems for an elastic plate. J. Math. Phys. 47(8), 12 (2006). (082901)

7. Cuccu, F.; Emamizadeh, B.; Porru, G.: Nonlinear elastic membranes involving the p-Laplacian operator. Electron. J. Differ. Equ. 49, 10 (2006)

8. Cuccu, F.; Porru, G.; Vitolo, A.: Optimization of the energy integral in two classes of rearrangements. Nonlinear Stud. 17(1), 23-35 (2010)

9. Del Pezzo, L.M.; Fernández Bonder, J.: Some optimization problems for $p$-Laplacian type equations. Appl. Math. Optim. 59(3), 365-381 (2009) 
10. Del Pezzo, L.M.; Fernández Bonder, J.: Remarks on an optimization problem for the $p$-Laplacian. Appl. Math. Lett. 23(2), 188-192 (2010)

11. Emamizadeh, B.; Zivari-Rezapour, M.: Rearrangements and minimization of the principal eigenvalue of a nonlinear Steklov problem. Nonlinear Anal. 74(16), 5697-5704 (2011)

12. Emamizadeh, B.; Zivari-Rezapour, M.: Rearrangement optimization for some elliptic equations. J. Optim. Theory Appl. 135(3), 367-379 (2007)

13. Emamizadeh, B.; Prajapat, J.V.: Maximax and minimax rearrangement optimization problems. Optim. Lett. 5(4), 647-664 (2011) 